\title{
Whole genome sequencing of Neisseria gonorrhoeae reveals transmission clusters involving patients of mixed HIV serostatus
}

\author{
Joanna Peters, ${ }^{1,2}$ Fiona Cresswell, ${ }^{3}$ Lauren Amor, ${ }^{4}$ Kevin Cole, ${ }^{1,2}$ Gillian Dean, ${ }^{3}$ \\ Xavier Didelot, ${ }^{5}$ Dilrini De Silva, ${ }^{6}$ David W Eyre, ${ }^{6}$ John Paul ${ }^{1,2}$
}

- Additional material is published online only. To view please visit the journal online (http://dx.doi.org/10.1136/ sextrans-2017-053198)

'Department of Microbiology, Public Health England Collaborative Centre, Royal Sussex County Hospital, Brighton, UK

${ }^{2}$ Department of Microbiology and Infection, Royal Sussex County Hospital, Brighton, UK ${ }^{3}$ Department of HIV and Sexual Health, Claude Nicol Centre, Royal Sussex County Hospital, Brighton, UK

${ }^{4}$ Brighton and Sussex Medical School, Brighton, UK

${ }^{5}$ Department of Infectious Disease Epidemiology, Imperial College London, London, UK

${ }^{6}$ Nuffield Department of Medicine, University of Oxford, Oxford, UK

Correspondence to Dr John Paul, Department of Microbiology and Infection, Royal Sussex County Hospital, Brighton BN2 5BE, UK; john. paul@phe.gov.uk

Received 20 March 2017 Revised 31 August 2017 Accepted 7 September 2017 Published Online First 11 October 2017

\begin{abstract}
Objectives Prevention and control of gonorrhoea depends on understanding the nature of sexual networks and risk factors for infection. We aimed to use highresolution typing (whole genome sequencing (WGS)) of Neisseria gonorrhoeae isolates plus patient questionnaire data to gain insights into transmission patterns in a high prevalence setting.
\end{abstract}

Methods During a 9-month period (July 2014March 2015), patients diagnosed with gonorrhoea attending sexual health service in Brighton, UK, were invited to provide anonymised detailed information by questionnaire about risk factors for infection. Questionnaire data plus WGS data from cultured isolates were analysed to yield information about sexual networks and risk factors for infection.

Results 104/149 individuals who consented to participate in the study were culture positive. 97/104 $(93 \%)$ were male. 80 self-reported to be men who have sex with men (MSM). 35/104 (34\%) of patients were HIV positive. 51/104 (49\%) individuals reported using geosocial networking applications to facilitate contact. Sex under the influence of drugs was reported by 16/34 (46\%) of HIV-positive MSM, 17/41 (41\%) of HIVnegative MSM and 5/15 (31\%) of heterosexuals. WGS data were available for 100 isolates from 83 patients. 55 isolates (66\%) belonged to genetically related subtypes involving one or more patients, who could be plausibly linked through recent direct or indirect transmission. Four transmission clusters containing 3-12 individuals were composed of MSM of mixed HIV serostatus.

Conclusions We show that data obtained from WGS of $N$. gonorrhoeae and enhanced epidemiological data obtained from patient questionnaires are mutually supportive and reveal insights into sexual networks. Our findings suggest that serosorting may have declined as a practice and indicate the importance of designing public health interventions that target infection risks associated with recreational drug use and contact made using geosocial networking applications.

\section{INTRODUCTION}

Gonorrhoea is of public health concern globally, with 78 million cases occurring annually and increasing rates of antimicrobial resistance. ${ }^{1}$ Between 2014 and 2015, the annual incidence rose in England by $11 \%$ from 37100 to 41193 reported cases. ${ }^{2}$ In the USA, almost 400000 cases were reported in 2015, a $12.8 \%$ increase from $2014 .^{3}$ Gonorrhoea is associated with significant morbidity including pelvic inflammatory disease and tubal factor infertility in women and epididymo-orchitis in men.

Gonorrhoea control depends on effective prevention, reliable diagnostics and prompt antimicrobial treatment of the index patient and recent sexual contacts. Asymptomatic carriage of Neisseria gonorrhoeae, especially in the rectum and pharynx, facilitates onward transmission, and therefore, frequent screening is recommended. For high-risk individuals, such as men who have sex with men (MSM), this is recommended every 3 months. ${ }^{4}$ However, despite evidence of increased access and engagement with sexual health services, gonorrhoea incidence is rising, particularly among MSM. ${ }^{3}$ Several possible drivers may explain this increase. First, the widespread use of online and mobile phone geosocial networking applications to facilitate contact may increase the number of casual sex partners and has been associated with STI outbreaks. ${ }^{5}$ Second, 'chemsex', which is associated with disinhibitory behaviour and prolonged episodes of condom-less sex with multiple partners, has become increasingly popular. $^{5-7}$ Third, concern about HIV acquisition may have diminished with increasing availability of HIV pre-exposure prophylaxis $(\mathrm{PrEP})^{8}$ and perceptions that effective antiretroviral therapy (ART) prevents onward transmission to HIV-negative partners. ${ }^{9}$

Understanding the networks within which gonorrhoea is transmitted is of crucial importance for directing public health interventions, where success depends on targeting effective interventions at appropriate individuals. $N$. gonorrhoeae multiantigen sequence typing has previously been used to identify strains associated with different populations but lacks the precision to link individuals, whereas whole genome sequencing (WGS) is more discriminatory and reveals networks of individuals infected with genetically related isolates. ${ }^{10} 11$ Combined with epidemiological data, WGS has the potential to shed light on local and global epidemiology of gonorrhoea.

We aimed to test the feasibility of using WGS data coupled with patient questionnaire data from a sexual health clinic to investigate and characterise transmission networks in a high prevalence setting. 


\section{METHODS}

\section{Setting and participants}

Brighton Sexual Health and Contraception centre has approximately 25000 attendances a year of which 25\% are MSM. In a prospective observational study all attendees, over a 9-month period (July 2014-March 2015, inclusive), with confirmed gonorrhoea on either nucleic acid amplification test (NAAT; BD ProbeTec, BD, Franklin Lakes, New Jersey, USA) or microscopy were invited to enter the study and complete a detailed questionnaire (see online supplementary materials 1 ) on sexual behaviour and lifestyle. Patients with gonorrhoea were treated with ceftriaxone plus azithromycin. Ethical approval was obtained from Brighton and Sussex Research Ethics Committee (14/LO/0435) to collect anonymised data from patients and invite them to complete a questionnaire. The questionnaire was developed in consultation with local practitioners with reference to the British National Survey of Sexual Attitudes and Lifestyles for rapid completion in the clinic to capture key information about infection risk factors. Individual consent for the use of anonymised bacterial isolates was not required.

\section{Samples}

Urogenital, rectal or pharyngeal samples were obtained as part of routine sexual health testing. Study isolates of N. gonorrhoeae (VCAT selective-agar, Oxoid, Basingstoke, UK) at Royal Sussex County Hospital laboratory underwent WGS as part of a previously published study. ${ }^{10}$

\section{Procedures}

Laboratory and sequencing procedures have been previously described in detail. ${ }^{10}$ Samples were processed in a routine diagnostic laboratory where isolates were identified and antibiotic susceptibilities were determined. A sweep of colonies from culture-positive selective agar plates underwent Illumina (San Diego, California, USA) WGS. Sequence data were mapped to the N. gonorrhoeae NCCP11945 reference genome and variants identified and compared with single nucleotide polymorphisms (SNPs) obtained from maximum likelihood phylogenetic trees, adjusted from the impact of recombination with ClonalFrameML. ${ }^{12}$ Sequence data (see online supplementary materials) were deposited at the National Center for Biotechnology Information. Isolates were assigned to subtypes, related by recent direct or indirect transmission, using a transmission nomogram, described elsewhere, ${ }^{10}$ taking into account genetic relatedness of isolates as well as sampling dates. The nomogram gives the expected range in genetic difference (SNPs) between isolates that includes $99 \%$ of direct or indirect transmissions for a given time (eg, 0-11 SNPs for samples taken 6 months apart). When identifying transmission clusters, all previously sequenced isolates were considered, including those without questionnaire data; however, only isolates from patients with questionnaire data are depicted in the current study. Sequenced isolates from the same patient that were not related by transmission using the transmission nomogram were considered to be distinct infections.

Consenting individuals completed a two-page questionnaire with information about their sexual history, condom use, STI history, use of internet/mobile phone apps to facilitate sex, travel history, recreational drug and alcohol consumption. Data collected were analysed using IBM SPSS Statistics (V.22).

\section{RESULTS}

Three-hundred and thirty-nine patients were NAAT-positive for N. gonorrhoeae during the study period. One hundred and forty-nine $(44 \%)$ individuals consented to participate in the study. Forty-five of 149 (30\%) patients were culture-negative. Questionnaire data were analysed for the 104/149 (70\%) individuals with a positive $N$. gonorrhoeae culture. Ninety-seven of 104 (93\%) of patients were male, and 90/104 (87\%) were white. Median age was 35 years old (range 20-61 years), and the most represented age group was 25-44 years (70\%). Thirty-five of $104(34 \%)$ of patients were HIV-positive. Sexual orientation was: MSM: 80/104 (77\%), bisexual: 6/104 (6\%), heterosexual: 15/104 (14\%) and unreported: 3/104 (3\%). WGS data were available, from a previous transmission study, ${ }^{10}$ for 100 isolates from $83 / 104(80 \%)$ patients.

\section{Sexual behaviour}

The median number of sexual partners in the past 3 months was 1 (range: 1-23) for heterosexuals, 5 (range: 1-45) for HIV-positive MSM and 5 for HIV-negative MSM (range 1-30). Fifty-one of 104 (49\%) individuals reported using geosocial networking applications to facilitate contact with partners. Overall, condom usage in the preceding 3 months was reported as: always by 16/86 (19\%), sometimes 47 (54\%) and never by 18 (21\%).

\section{Recreational drug use}

Sex under the influence of drugs was reported by $16 / 34$ (46\%) of HIV-positive MSM, 17/41 (41\%) of HIV-negative MSM and $5 / 15(33 \%)$ of heterosexuals. Mephedrone and cocaine were frequently cited by MSM and heterosexuals, respectively. Ten (six HIV-positive) MSM reported 'slamming' (injecting drugs at the time of sex) in the previous 3 months.

\section{Site of infection}

Forty-five of 104 (43\%) individuals had rectal infection, 20/104 (19\%) had pharyngeal infection, 53/104 (51\%) had urethral infection and 7/104 (7\%) had cervical infection. Many individuals were simultaneously infected at multiple sites. Sites of infection in MSM are shown in table 1.

\section{Genomic analysis}

From 100 sequenced isolates, we identified 84 genetically distinct infections in 83 patients, caused by 44 distinct genetic subtypes of $N$. gonorrhoeae, where isolates within a subtype were related by direct (person-to-person) or indirect (via $\geq 1$ intermediate source) transmission (figure 1). Twenty-eight subtypes were identified in a single infected patient and could not be linked to others within this particular data set. The remaining 56 infections $(67 \%)$ belonged to subtypes represented by 14 clusters of two or more isolates where infected patients could be plausibly linked to one or more others. Patient characteristics and behavioural information for these clusters are given in table 2 .

\begin{tabular}{lc}
\hline Table 1 & Anatomical sites of infection in MSM \\
\hline Site & Number of patients \\
\hline Rectum only & 31 \\
Urethra only & 30 \\
Throat only & 11 \\
Rectum+urethra & 7 \\
Rectum+throat & 4 \\
Rectum+urethra+throat & 1 \\
Urethra+throat & 2 \\
Total & 86 \\
\hline
\end{tabular}

MSM, men who have sex with men. 
HIV positive (MSM)

HIV negative (MSM)

- HIV negative (Heterosexual)

- HIV negative (Sexual orientation not recorded)

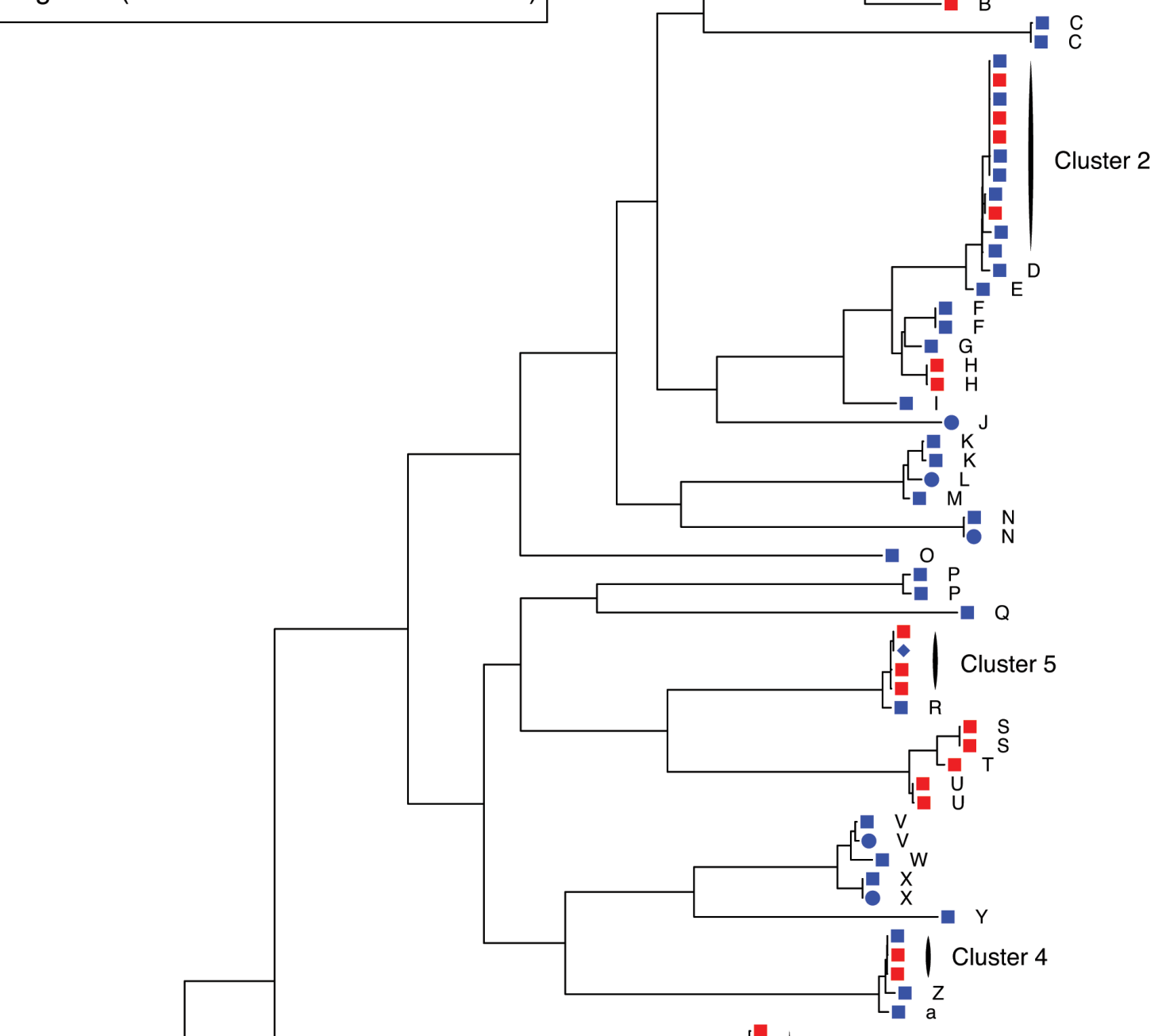

Cluster 1

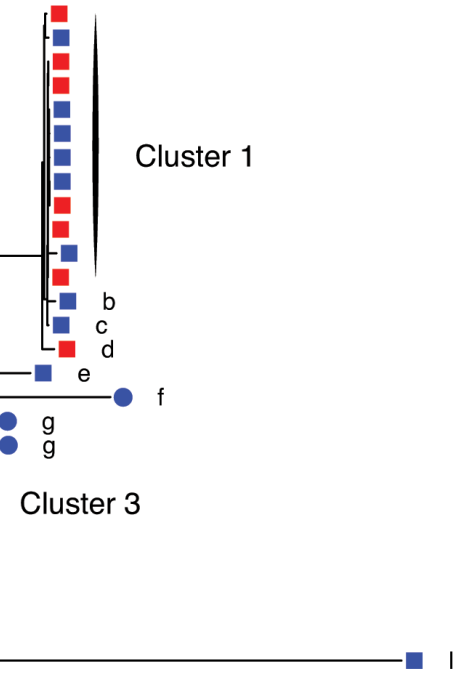

\section{0 single nucleotide polymorphisms}

Figure 1 Maximum likelihood recombination-corrected phylogenetic tree of 84 genetically distinct infections identified in 83 individuals with whole genome sequencing data and questionnaire data. The tips of the tree are labelled with transmission cluster identifiers (A-Z, a-m) based on transmission clusters identified in De Silva et al (2016). ${ }^{10}$ Five clusters with three or more cases with questionnaire data are highlighted. A single isolate for each patient infection is shown. MSM, men who have sex with men. 
Table 2 Patient characteristics and behavioural information for 14 clusters of patients linked by WGS data

\begin{tabular}{|c|c|c|c|c|c|c|c|c|c|c|c|c|c|c|}
\hline Cluster & 1 & 2 & 3 & 4 & 5 & 6 & 7 & 8 & 9 & 10 & 11 & 12 & 13 & 14 \\
\hline Number of patients & 12 & 11 & 4 & 3 & 4 & 2 & 2 & 2 & 2 & 2 & 2 & 2 & 2 & 2 \\
\hline MSM/bisexual:heterosexual & 12:0 & $11: 0$ & $1: 3$ & $3: 0$ & $3: 0^{*}$ & $2: 0$ & $2: 0$ & $2: 0$ & $2: 0$ & $2: 0$ & $2: 0$ & $2: 0$ & $2: 0$ & $2: 0$ \\
\hline HIV-positive: HIV-negative & $6: 6$ & $4: 7$ & $0: 4$ & $2: 1$ & $3: 1$ & $0: 2$ & $0: 2$ & $2: 0$ & $0: 2$ & $0: 2$ & $0: 2$ & $2: 0$ & $2: 0$ & $2: 0$ \\
\hline Reported chemsex & $5 / 12$ & $7 / 11$ & $1 / 4$ & $1 / 3$ & $3 / 4$ & $1 / 2$ & $0 / 2$ & $0 / 2$ & $2 / 2$ & $0 / 2$ & $0 / 2$ & $1 / 2$ & $0 / 2$ & $1 / 2$ \\
\hline Reported mobile phone app use & $5 / 12$ & $6 / 11$ & $2 / 4$ & $2 / 3$ & $3 / 4$ & $2 / 2$ & $2 / 2$ & $2 / 2$ & $1 / 2$ & $0 / 2$ & $1 / 2$ & $1 / 2$ & $1 / 2$ & $2 / 2$ \\
\hline Reported condomless sex & $7 / 12$ & $8 / 11$ & $3 / 4$ & $3 / 3$ & $3 / 4$ & $2 / 2$ & $1 / 2$ & $2 / 2$ & $2 / 2$ & $2 / 2$ & $2 / 2$ & $2 / 2$ & $1 / 2$ & $2 / 2$ \\
\hline
\end{tabular}

*One patient did not report sexual orientation.

MSM, men who have sex with men; WGS, whole genome sequencing.

\section{Cluster 1}

The largest cluster (figure 2) comprised 12 MSM, of whom six were HIV-positive and six HIV-negative. Five individuals were infected at more than one anatomical site: rectum $(n=9)$, urethra $(\mathrm{n}=5)$ and pharynx $(\mathrm{n}=3)$. One individual in this cluster with rectal infection yielded a genetically unrelated strain from a urethral sample and belonged simultaneously to two separate transmission networks. Individual sexual practice was diverse, with the majority practising insertive (9/12) and receptive $(10 / 12)$ anal intercourse and active (11/12) and receptive fellatio (10/12). Five of 12 patients reported chemsex (defined as use of $\gamma$-hydroxybutyrate, 4-methylmethcathinone or methamphetamine to facilitate or enhance sex), including one individual who reported injecting drug use at the time of sex. Condom use was recorded as: always by $4 / 12$, sometimes by $4 / 12$, never by $3 / 12$ and unreported by $1 / 12$. To facilitate contact, $4 / 12$ had visited a sauna, 4/12 attended sex parties (4/12) and 5/12 used mobile phone apps. During the preceding 3 months, seven individuals reported sex outside Brighton: London (5/12), Spain (1/12) and Germany (1/12); the remainder reported sex only in Brighton.

\section{Cluster 2}

The second largest cluster (figure 2) comprised four HIV-positive MSM and seven HIV-negative MSM. Three were infected at more than one anatomical site. Infection sites were: urethra $(\mathrm{n}=6)$, rectum $(\mathrm{n}=5)$ and pharynx $(\mathrm{n}=3)$. Sexual practice was diverse with the majority practising receptive (8/11) and insertive $(10 / 11)$ anal intercourse, and active and receptive fellatio (11/11). Condom use was reported as: always by $3 / 11$, sometimes by $6 / 11$ and never by $2 / 11$. Six of 11 reported use of mobile phone apps to facilitate sex, $3 / 11$ had visited saunas and 6/11 had attended sex parties. Seven of 11 reported chemsex (including slamming by one individual).

\section{Cluster 3}

A smaller cluster (figure 2) comprised three self-reported heterosexual males and one MSM; all HIV negative with urethral infection. Two of 4 individuals reported using mobile phone apps. The heterosexual males reported insertive vaginal intercourse and active and receptive oral sex. The MSM reported insertive and receptive anal and oral intercourse. Chemsex was reported only by the MSM. Condom use was recorded as: never by $1 / 4$,
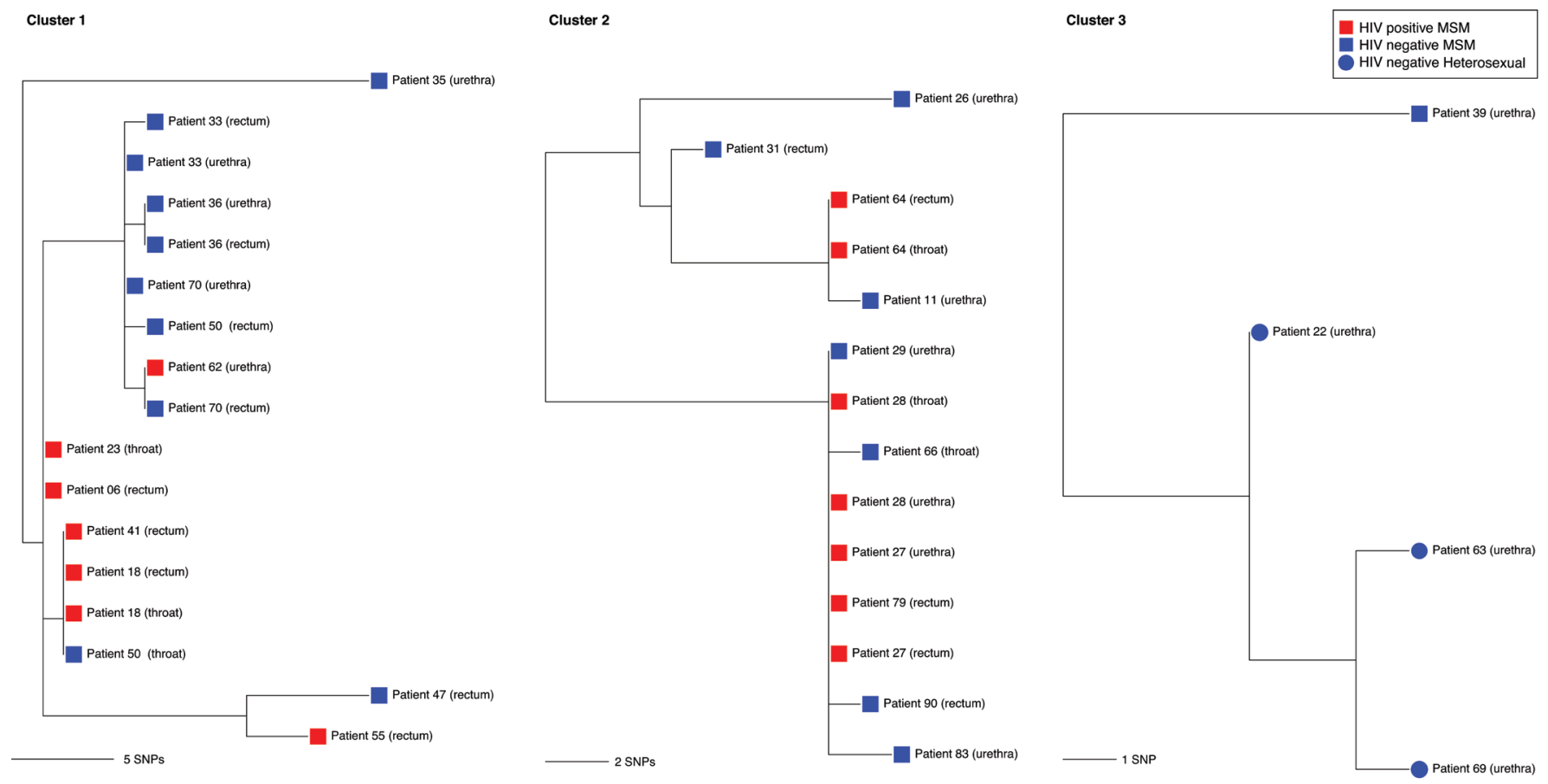

HIV positive MSM HIV negative MSM HIV negative Heterosexual

Figure 2 Maximum likelihood recombination-corrected phylogenetic trees of transmission clusters 1-3. Where more than one sequence was available from a patient, all are shown. MSM, men who have sex with men; SNPs, single nucleotide polymorphisms. 
sometimes by $2 / 4$ and unreported by $1 / 4$. The MSM reported sex in Manchester and the USA.

Two smaller clusters (cluster 4, n=3) and cluster 5, n=4) both comprised individuals of mixed HIV serostatus (figure 1). Most of these individuals reported mobile phone app use to make contact and chemsex.

\section{DISCUSSION}

We used WGS and patient questionnaire data to investigate gonorrhoea transmission in Brighton over a 9-month period. Even within this relatively small study sample, most infections $(56 / 84,67 \%)$ could be plausibly linked to another case through recent direct or indirect transmission. Unlinked cases in our study can be explained by patients who did not consent to provide questionnaire data, culture-negative patients and sources of infection outside Brighton. Some highly mobile individuals have sexual encounters and seek sexual healthcare in other cities and countries. As reported elsewhere, WGS has demonstrated transmission links between Brighton, other regions of the UK and the USA, ${ }^{10}$ highlighting the need for gonorrhoea control efforts to span regional and national boundaries.

Brighton has a large MSM population and relatively high rates of gonorrhoea and also HIV. We found clusters of plausibly linked gonorrhoea cases that included patients of mixed HIV serostatus. Although some of our participants may have selected partners according to HIV status, our results provide evidence that serosorting was not consistently practised. This may be related to lower risk thresholds associated with chemsex. ${ }^{5}$ Another explanation is use of HIV PrEP: a PrEP effectiveness study (Pre-exposure prophylaxis to prevent the acquisition of HIV-1 infection: PROUD) $)^{13}$ was in progress in Brighton during the study period. Additionally, individuals may feel less motivated to use a condom with a serodiscordant partner if the HIV-positive partner is receiving ART. The partner study reported no linked HIV transmission in HIV serodiscordant couples during 16400 episodes of sex where the HIV-positive partner received ART and viral load was $<200$ copies/mL. ${ }^{9}$ We did not collect data on strategic positioning, which has been reported to be a common practice in highly sexually active HIV-negative MSM. ${ }^{14}$ We also found evidence of bridging of infection between heterosexuals and MSM. One cluster (cluster 3 ) included highly related (2-3 SNPs) isolates from three heterosexual men and one isolate from an MSM. The MSM isolate differed by nine SNPs from the heterosexual subcluster, suggesting the existence of missing links with the heterosexual group.

Our results suggest that WGS of pathogens can be used to support conventional epidemiology in defining and characterising core transmitters ${ }^{15} 16$ and allow better targeted and more

Key messages

- Combined whole genome sequencing data and patient questionnaire data allows enhanced understanding of gonorrhoea transmission.

- Gonorrhoea transmission networks include patients of mixed HIV serostatus: serosorting may be declining as a practice.

- Public health interventions should be designed to minimise the risk of disease transmission associated with use of recreational drugs ('chemsex') and use of geosocial networking applications to facilitate contact with partners. effective control efforts to reduce transmission. Our approach allows identification of risk factors, one being the use of mobile geosocial networking applications to facilitate contact. Using phone apps to transmit prevention messages, STI testing information and to undertake partner notification should be explored. Educational messages and substance misuse services focused on chemsex may also have the potential to reduce frequency of partner change and risk taking behaviour. Public Health England released a briefing document for commissioners and providers of drug and alcohol services containing information about chemsex. ${ }^{17}$ New HIV diagnoses in the over 50s have doubled over the last decade. ${ }^{18}$

One individual had two distinct $N$. gonorrhoeae strains, with different antimicrobial resistance patterns, in different anatomical sites suggesting they were part of two distinct transmission chains. This is consistent with the previous finding, based on WGS, that $13 \%$ of Brighton patients had distinct infections at different anatomical sites, ${ }^{10}$ and highlights the importance of triple site testing, as well as antimicrobial resistance testing, in MSM.

We aimed to test the feasibility of using WGS data coupled with patient questionnaire data to characterise sexual networks. A limitation is that we did not formally investigate congruence of WGS-defined clusters with partner notification records. Nevertheless, WGS and epidemiological results are broadly mutually supportive. Our findings may not be generalisable to other locations, especially where gonorrhoea occurs more commonly in heterosexual populations. Another limitation is that we obtained both WGS data and questionnaire data from only a subset of patients presenting with gonorrhoea. However, we believe selection bias may be minimal as the demographics of the study population correlate with the demographics of the general population experiencing gonorrhoea in Brighton based on local Genito-Urinary Medicine Clinic Activity Dataset ${ }^{19}$ data. It is possible that participants may have under-reported their risk-taking behaviour, such as slamming, sex parties use of commercial sex workers, which may lead to a reporting bias, although to minimise this patients completed the questionnaires themselves anonymously.

This study raises questions about whether HIV serosorting has declined as a practice and why this might be. It has highlighted common risk factors such as chemsex and mobile phone apps to facilitate sex among MSM, both of which could be suitable targets for tailored interventions. We also found evidence of bridging between populations: it is plausible that interventions that reduce transmission in MSM may have wider impact in the general population. Genome sequencing is becoming increasingly accessible and offers opportunities to improve the investigation and control of gonorrhoea.

\section{Handling editor Catherine $A$ Ison}

Acknowledgements The authors would like to thank Angela Dunn and Andrew Bexley for help with patient recruitment.

Contributors JPe, FC, GD, DDS, DWE and JPa designed the study. JPe, FC, LA and GD collected patient data and isolates. KC processed the isolates. XD, DDS and DWE did bioinformatic analysis. JPe, FC, LA, XD, DWE and JPa analysed the data. DWE prepared the figures. JPe, FC, LA, DWE and JPa wrote the manuscript, which was revised by all authors.

Funding The research was funded by the National Institute for Health Research Health Protection Research Unit (NIHRHPRU) in Healthcare Associated Infections and Antimicrobial Resistance at the University of Oxford in partnership with Public Health England (PHE) (HPRU-2012-10041) and the NIHR HPRU in Modelling Methodology at Imperial College London (HPRU-2012-10080) in partnership with PHE.

Competing interests DWE is an NIHR clinical lecturer.

Ethics approval Brighton and Sussex Research Ethics Committee (14/LO/0435). 
Provenance and peer review Not commissioned; externally peer reviewed.

(c) Article author(s) (or their employer(s) unless otherwise stated in the text of the article) 2018. All rights reserved. No commercial use is permitted unless otherwise expressly granted.

\section{REFERENCES}

1 Newman L, Rowley J, Vander Hoorn S, et al. Global estimates of the prevalence and incidence of four curable sexually transmitted infections in 2012 based on systematic review and global reporting. PLoS One 2015;10:e0143304.

2 Anon. Sexually transmitted infections and chlamydia screening in England. Health Protection Report 2016:10:27.

3 Anon. Sexually transmitted diseases surveillance. Centers for disease control and prevention. 2015 https://www.cdc.gov/std/stats15/gonorrhea.htm (accessed 20 Mar 2017).

4 Clutterbuck DJ, Flowers P, Barber T, et al. UK national guideline on safer sex advice. Int J STD AIDS 2012;23:381-8.

5 Gilbart VL, Simms I, Jenkins C, et al. Sex, drugs and smart phone applications: findings from semistructured interviews with men who have sex with men diagnosed with Shigella flexneri 3a in England and wales. Sex Transm Infect 2015;91:598-602.

6 Thomas DR, Williams CJ, Andrady U, et al. Outbreak of syphilis in men who have sex with men living in rural North Wales (UK) associated with the use of social media. Sex Transm Infect 2016;92:359-64.

7 Payne L, Lawrence D, Soni S, et al. Investigating factors for increased gonorrhoea reinfection in men who have sex with men attending a genitourinary clinic: a qualitative study. Int J STD AIDS 2017;28:858-63.

8 Kojima N, Davey DJ, Klausner J. Meta-analysis finds association between pre-exposure prophylaxis to prevent Human Immunodeficiency Virus (HIV) infection and increased risk of sexually transmitted infection acquisition among men who have sex with men. Open Forum Infect Dis 2016;3:504.
9 Rodger AJ, Cambiano V, Bruun T, et al. Sexual activity without condoms and risk of HIV transmission in serodifferent couples when the HIV-positive partner is using suppressive antiretroviral therapy. JAMA 2016;316:171-81.

10 De Silva $\mathrm{D}$, Peters J, Cole K, et al. Whole-genome sequencing to determine transmission of Neisseria gonorrhoeae: an observational study. Lancet Infect Dis 2016;16:1295-303.

11 Didelot X, Dordel J, Whittles LK, et al. Genomic analysis and comparison of two gonorrhea outbreaks. MBio 2016;7:e00525-16.

12 Didelot $X$, Wilson DJ. ClonalFrameML: efficient inference of recombination in whole bacterial genomes. PLoS Comput Biol 2015;11:e1004041.

13 McCormack S, Dunn DT, Desai M, et al. Pre-exposure prophylaxis to prevent the acquisition of HIV-1 infection (PROUD): effectiveness results from the pilot phase of a pragmatic open-label randomised trial. Lancet 2016;387:53-60.

14 Grov C, Rendina HJ, Moody RL, et al. HIV serosorting, status disclosure, and strategic positioning among highly sexually active gay and bisexual men. AIDS Patient Care STDS 2015;29:559-68.

15 Thomas JC, Tucker MJ. The development and use of the concept of a sexually transmitted disease core. J Infect Dis 1996;174 Suppl 2:S134-S143.

16 Yorke JA, Hethcote HW, Nold A. Dynamics and control of the transmission of gonorrhea. Sex Transm Dis 1978;5:51-6.

17 Anon. Substance misuse services for men who have sex with men involved in chemsex, 2015. PHE publications gateway number: 2015476 November.

18 Mahy M, Autenrieth CS, Stanecki K, et al. Increasing trends in HIV prevalence among people aged 50 years and older: evidence from estimates and survey data. AIDS 2014;28 Suppl 4:S453-S459.

19 Anon. Genitourinary medicine clinic activity dataset (GUMCADv2): Public Health England, 2013. https://www.gov.uk/guidance/genitourinary-medicine-clinic-activitydataset-gumcadv2. 